\title{
Measuring Quality Improvement in Acute Ischemic Stroke Care: Interrupted Time Series Analysis of Door-to-Needle Time
}

\author{
Anne Margreet van Dishoeck ${ }^{a} \quad$ Diederik W.J. Dippel $^{b} \quad$ Maaike Dirks $^{b}$ \\ Caspar W.N. Looman ${ }^{a}$ Johan P. Mackenbach ${ }^{a}$ Ewout W. Steyerberg ${ }^{a}$ \\ Departments of a Public Health and ${ }^{b}$ Neurology, Erasmus MC, University Medical Center \\ Rotterdam, Rotterdam, The Netherlands
}

Key Words

Acute ischemic stroke Acute stroke care - Door-to-needle time $\cdot$ Recombinant tissue plasminogen activator - Performance indicator - Quality of care · Process indicators · Quality improvement

\section{Abstract}

Background: In patients with acute ischemic stroke, early treatment with recombinant tissue plasminogen activator (rtPA) improves functional outcome by effectively reducing disability and dependency. Timely thrombolysis, within $1 \mathrm{~h}$, is a vital aspect of acute stroke treatment, and is reflected in the widely used performance indicator 'door-to-needle time' (DNT). DNT measures the time from the moment the patient enters the emergency department until he/ she receives intravenous rtPA. The purpose of the study was to measure quality improvement from the first implementation of thrombolysis in stroke patients in a university hospital in the Netherlands. We further aimed to identify specific interventions that affect DNT. Methods: We included all patients with acute ischemic stroke consecutively admitted to a large university hospital in the Netherlands between January 2006 and December 2012, and focused on those treated with thrombolytic therapy on admission. Data were collected routinely for research purposes and internal quality measurement (the Erasmus Stroke Study). We used a retrospective interrupted time series design to study the trend in DNT, analyzed by means of segmented regression. Results: Between January 2006 and December 2012, 1,703 patients with ischemic stroke were admitted and 262 (17\%) were treated with rtPA. Patients treated with thrombolysis were on average 63 years old at the time of the stroke and $52 \%$ were male. Mean age $(p=0.58)$ and sex distribution $(p=0.98)$ did not change over the years. The proportion treated with thrombolysis increased from 5\% in 2006 to 22\% in 2012. In 2006, none of the patients were treated within $1 \mathrm{~h}$. In 2012, this had increased to 81\%. In a logistic regression analysis, this trend was significant (OR 1.6 per year, CI 1.4-1.8). The median DNT was re- 
duced from $75 \mathrm{~min}$ in 2006 to $45 \mathrm{~min}$ in 2012 ( $p$ < 0.001 in a linear regression model). In this period, a $12 \%$ annual decrease in DNT was achieved (CI from 16 to $8 \%$ ). We could not find a significant association between any specific intervention and the trend in DNT. Conclusion and Implications: The DNT steadily improved from the first implementation of thrombolysis. Specific explanations for this improvement require further study, and may relate to the combined impact of a series of structural and logistic interventions. Our results support the use of performance measures for internal communication. Median DNT should be used on a monthly or quarterly basis to inform all professionals treating stroke patient of their achievements.

(C) 2014 S. Karger AG, Basel

\section{Background}

In patients with acute ischemic stroke, early treatment with recombinant tissue plasminogen activator (rtPA) improves functional outcome by effectively reducing disability and dependency $[1,2]$. Recent guidelines for the treatment of ischemic stroke recommend that the time from arrival at the hospital to the initiation of the thrombolytic treatment should be $1 \mathrm{~h}$ or less [3]. The quality of the in-hospital care pathway is often measured by means of the time from the moment the patient enters the emergency department (ED) until he/she receives intravenous rtPA: the door-to-needle time (DNT). Intraorganizational barriers to timely thrombolysis relate to the availability of a neurologist, blood drawing and measurements, computed tomographic (CT) imaging and skilled nursing staff $[4,5]$. In the Netherlands, the percentage of patients receiving thrombolysis within $1 \mathrm{~h}$ is a mandatory indicator of hospital performance for external accountability.

In a large university hospital in the Netherlands, stroke care has been guided by a hospitalwide protocol since 2001. The neurology department implemented several quality initiatives to improve the care for acute stroke patients, especially focusing on the percentage of patients receiving thrombolysis [6, 7]. Improving DNT started in 2005 with a yearly training of residents and nursing staff, including 'dummy runs'. Pocket flowcharts with protocol summaries were first handed out in 2006 and were updated regularly. In July 2007, the ED initiated the use of the Manchester Triage System (MTS) protocol. The MTS is a sensitive tool for marking those who need critical care on arrival in the ED. Stroke patients obtain the highest emergency code red [8]. From October 2007 onwards, treatment was started in the CT room, and DNT was reported for every patient at the morning report. Individual feedback was given to all doctors who exceeded the 1-hour time threshold. In October 2009, a CT scanner was placed in the ED and treatment started immediately after noncontrast CT imaging was done, but before CT angiography. In November 2010, a prenotification single-call activation system was put in place, alarming the neurology resident, radiologist, radiology laboratory personnel and the ED nurse. In May 2011, it was decided to have a second neurology resident on duty during the weekends to ensure the availability of a doctor at any time.

We aimed to study quality improvement from the first implementation of thrombolysis in this university hospital. We further aimed to identify specific interventions that affect DNT.

\section{Methods}

We included all acute ischemic stroke patients admitted to a large university hospital in the Netherlands between 2006 and 2012. We focused on those treated with thrombolytic therapy on admission. A retrospective interrupted time series design was used to evaluate 
longitudinal effects [9]. Segmented regression analysis of the interrupted time series data allowed us to assess how much an intervention changed the DNT. The time series experiment is a reasonable alternative when the condition of a true experiment cannot be met [9].

Data were collected routinely for research purposes and internal quality measurement (the Erasmus Stroke Study) and for the reporting of the performance indicator 'timely thrombolysis' in ischemic stroke patients. All patients with acute stroke admitted to the neurology department were entered into the registry. Completeness was cross-checked with hospital administrative systems. Data were entered and checked by medical researchers. They were anonymized for analysis and could not be related to individual patients. The data collection did not change over time. The Erasmus Stroke Study has been approved for use in scientific medical studies by the institutional review board of Erasmus MC. We selected quality interventions that had a fixed starting point in time to include in the model. Selected interventions (i) were the start of the educational program (i1), MST protocol (i2), CT scanner at the ED (i3), prenotification system (i4) and second neurology resident (i5).

We report descriptive statistics using percentages, means and standard deviations or medians and interquartile ranges. We estimated the trend in DNT from the start of the measurement in 2006 and tested for changes in the dependent variable 'before and after intervention' with a segmented regression analysis. Two models were considered. The first model was: $\log (\mathrm{DTN})=\alpha+\beta_{\mathrm{T}} \mathrm{T}$. The second model was: $\log (\mathrm{DTN})=\alpha+\beta_{\mathrm{T}} \mathrm{T}+\sum_{1-5}\left[\beta_{\mathrm{i}} \mathrm{I}_{\mathrm{i}}\right]+\sum_{1-5}$ $\left[\beta_{\mathrm{i} \times \mathrm{T}} \mathrm{I}_{\mathrm{T}} \times \mathrm{T}\right]$, where $\mathrm{T}$ (time) represents the time from the start of the measurement period (continuous variable, months starting at 1 ), and $\beta_{\mathrm{T}}$ expresses the overall trend before the interventions. I (intervention) represents the difference between before and after intervention (i), coded 0 for prior to the intervention and 1 for after the intervention; $\beta_{\mathrm{i}}$ expresses the drop in DNT immediately after an intervention, and $\beta_{\mathrm{i} \times \mathrm{T}}$ indicates the change in trend over time. Both models were fitted with and without inclusion of potential confounders (age and sex). The estimate $\left(\mathrm{e}^{\beta}-1\right) \times 100$ represents the percentage change in DNT. The confidence interval was calculated as $100 \times\left[\mathrm{e}^{\beta} \pm(1.96 \times \mathrm{SE})-1\right]$, where SE is the standard error for the $\beta$ parameter considered. We additionally performed a logistic regression analysis to estimate change over time in the percentage of patients receiving thrombolysis within $1 \mathrm{~h}$. Statistical analysis was done with IBM SPSS statistics v. 20 and R v. 3.0.1 (R Foundation for Statistical Computing, Vienna, Austria).

\section{Results}

Between January 2006 and December 2012, 1,703 patients with ischemic stroke were admitted and $285(17 \%)$ were treated with rtPA. We excluded 17 patients because of referral from another hospital for intra-arterial thrombolysis, 3 patients because of an in-hospital event and 3 patients because of missing data, leaving 262 patients. Patients treated with thrombolysis were on average 63 years old at the time of the stroke and $52 \%$ were male (table 1). Mean age $(\mathrm{p}=0.58)$ and sex distribution $(\mathrm{p}=0.98)$ did not change over the years. The proportion treated with thrombolysis increased from 5\% in 2006 to $22 \%$ in 2012 (fig. 1). In 2006, none of the patients were treated within $1 \mathrm{~h}$. In 2012, this had increased to $81 \%$ (fig. 1). In a logistic regression analysis this trend was significant (OR 1.6 per year, CI 1.4-1.8). The median DNT was reduced from $75 \mathrm{~min}$ in 2006 to $45 \mathrm{~min}$ in 2012 ( $\mathrm{p}<0.001$, in a linear regression model). In this period, a 12\% annual decrease in DNT was achieved (CI from 16 to 8\%). We could not find a significant association between any specific intervention (fig. 2) and the trend in DNT. 


\section{Cerebrovascular' \\ Diseases}

Fig. 1. Percentage of patients with acute ischemic stroke treated with thrombolysis (red/green bars) and the fraction treated within $1 \mathrm{~h}$ (green) per year from 2006 to 2012.

\begin{tabular}{l|l}
\hline Cerebrovasc Dis Extra 2014;4:149-155 \\
\hline DOI: $10.1159 / 000363535$ & $\begin{array}{l}\text { (c) 2014 S. Karger AG, Basel } \\
\text { www.karger.com/cee }\end{array}$ \\
\hline
\end{tabular}

van Dishoeck et al.: Measuring Quality Improvement in Acute Ischemic Stroke Care: Interrupted Time Series Analysis of Door-to-Needle Time

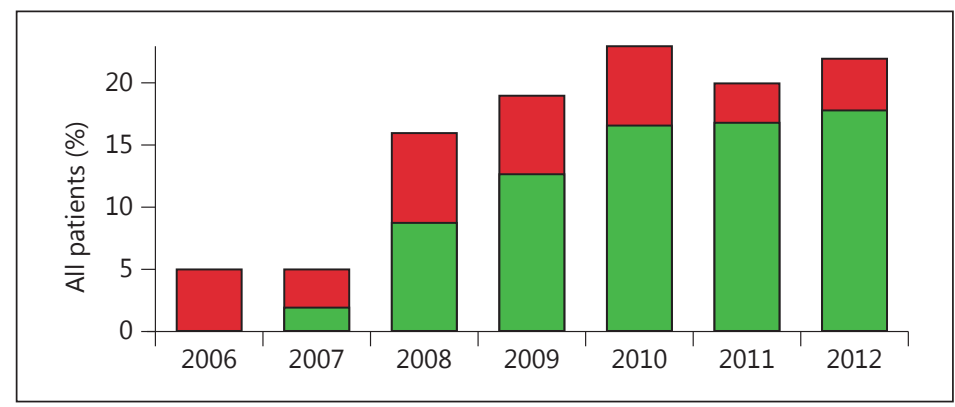

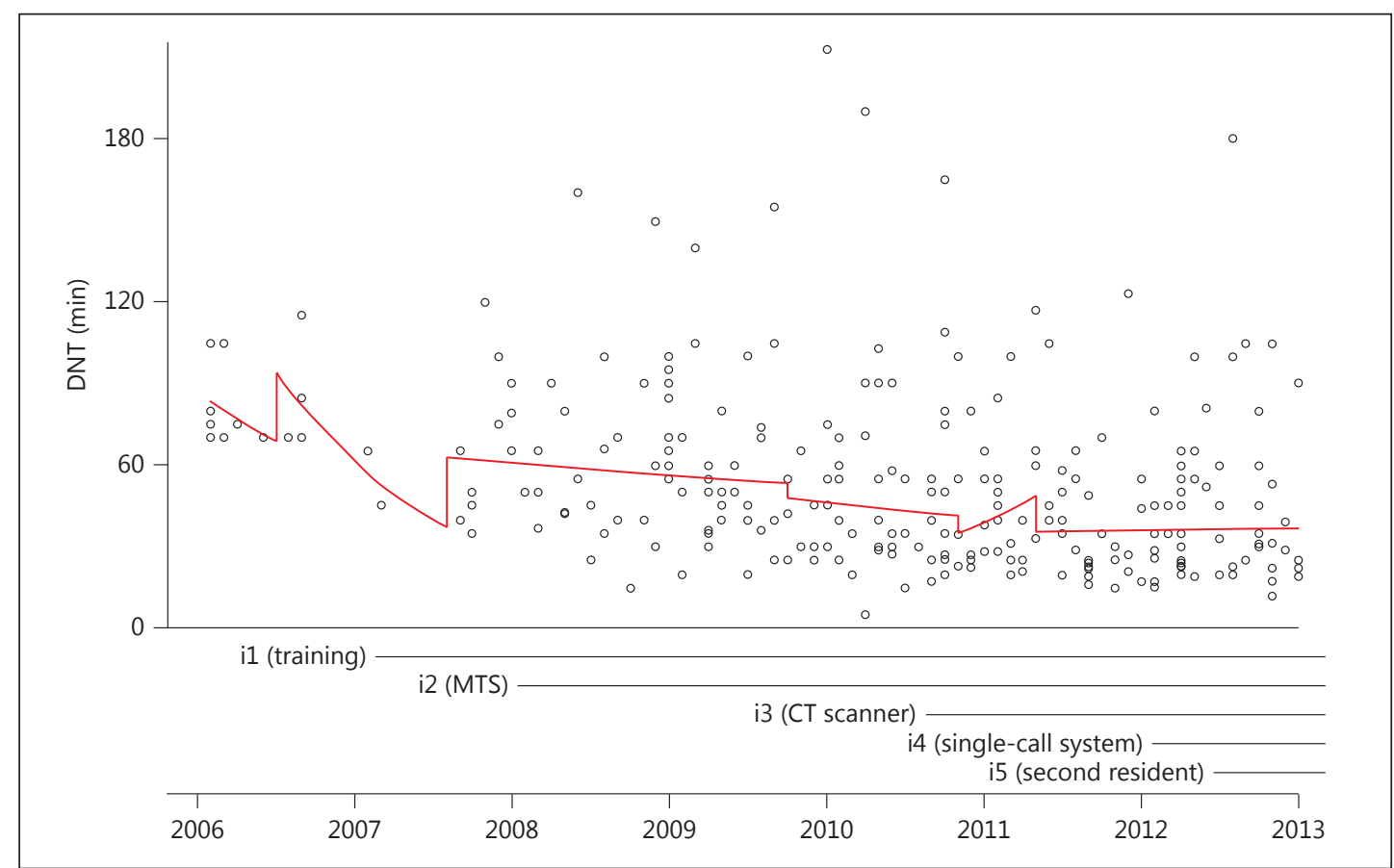

Fig. 2. Scatter plot with regression lines indicating effects of interventions aimed at improving DNT for acute ischemic stroke treatment with rtPA.

Table 1. Demographic characteristics

\begin{tabular}{lcllrl}
\hline Year & $\begin{array}{l}\text { Admissions to } \\
\text { hospital for } \\
\text { ischemic stroke, } \mathrm{n}\end{array}$ & $\begin{array}{l}\text { Thrombolysis } \\
\text { after admission } \\
\text { to ED, } \mathrm{n}\end{array}$ & Mean age $^{1} \pm \mathrm{SD}$ & $\begin{array}{l}\text { Gender }^{1} \\
\text { (male), } \mathrm{n}\end{array}$ & Median DNT \\
\hline 2006 & 262 & $12(5)$ & $60 \pm 14.9$ & $6(50)$ & $75(70-100)$ \\
2007 & 266 & $13(5)$ & $60 \pm 17.2$ & $8(62)$ & $65(45-85)$ \\
2008 & 200 & $31(16)$ & $67 \pm 16.5$ & $15(48)$ & $60(42-90)$ \\
2009 & 232 & $45(19)$ & $62 \pm 17.4$ & $25(56)$ & $50(36-72)$ \\
2010 & 235 & $54(23)$ & $62 \pm 16.1$ & $27(51)$ & $40(27-68)$ \\
2011 & 242 & $49(20)$ & $65 \pm 14.7$ & $26(53)$ & $40(25-55)$ \\
2012 & 261 & $58(22)$ & $63 \pm 15.8$ & $29(50)$ & $35(23-56)$ \\
Total & 1,703 & 262 & $63 \pm 16.1$ & $136(52)$ & $45(30-70)$ \\
\hline
\end{tabular}

Figures in parentheses are percentages or interquartile ranges.

${ }^{1}$ Age and gender of the patients are related to rtPA treatment. 

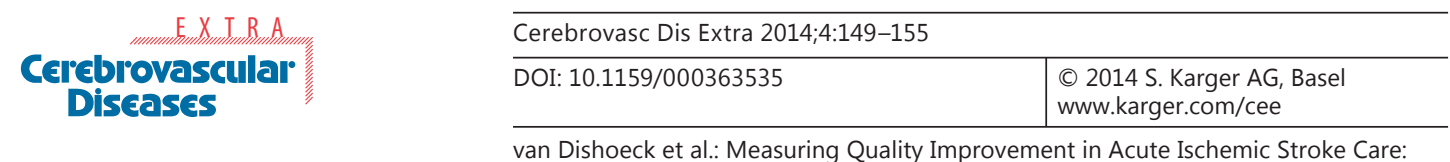

van Dishoeck et al.: Measuring Quality Improvement in Acute Ischemic Stroke Care Interrupted Time Series Analysis of Door-to-Needle Time

\section{Discussion}

The percentage of patients treated within $1 \mathrm{~h}$ increased from 0 to $81 \%$. This increasing trend is observed in several other studies [10-12].

We found that the median DNT was successfully reduced by 30 min between 2006 and 2012. Although the DNT improved significantly, we could not attribute this trend to one or more specific interventions. We note that all implemented interventions have been proven effective in the literature [3, 5, 13-22]. An explanation for the lack of significance in our analysis may lie in a slow and gradual effect of our interventions. We selected only those interventions with a fixed starting point in time for our analysis. Other initiatives, such as discussing the DNT for every patient at the morning report, could also explain the reduction in DNT (residual confounding). We hypothesize that the cumulative effect of various interventions lowered the DNT. The constant and increasing focus on improvement may have steered the perception of urgency among physicians and ED personnel. Such a perception might translate into faster action to initiate treatment $[5,23]$. This highlights the complexity of quality improvement within a single-center setting and of relating the results to a single measurement. A recent review evaluated the effectiveness of improvements from quality collaboratives, especially feedback systems [24]. It concluded that although the evidence of the impact of quality collaboratives is positive, it is also limited because of the complex nature of improvements and the different ways they are applied. Our results resemble those of Meretoja et al. [25], who reduced the median in-hospital delay to 20 min with multiple concurrent strategies, but they did not relate the decrease of DNT to any single intervention. It hence remains unclear what specific mechanisms or interventions are responsible for the quality improvement.

We assumed that the groups of patients were similar every year. We could check this assumption for age and gender and stroke severity, which were similar over the years and did not affect the findings. Other, unmeasured, confounders may however have influenced the results. We speculate that the increase in the proportion of patients treated with rtPA over the years means that more complicated patients were also treated, for example those with not readily available information on contraindicated medication, or with fluctuating symptoms or high blood pressure. This implies that the observed trend in decreased DNT would even have been stronger if the same selection of patients had been made as in the early years of rtPA treatment.

Our results support the use of performance measures for internal communication. Median DNT should be used on a monthly or quarterly basis to inform all professionals treating stroke patient of their achievements. Measuring and reporting DNT could be helpful in keeping professionals focused and in improving performance [15].

Limitations of our research are the single-center design without a control group and a relatively small sample size. The small sample size may explain our lack of statistically significant results for specific interventions. It implies that scientifically valid evaluations of local implementation are only possible in large centers with large caseloads. The lack of effect of specific interventions may be explained by a type 2 error (lack of power) or by a true absence. It remains unclear if the performance indicator is not suitable for explaining the individual interventions (type 2 error or lack of power) in the single-center setting or if the intervention itself did not have a major impact. Furthermore, we did not focus on measuring more specific parts of the care processes, like 'onset to door time', 'door-to-CT time' or 'door-to-neurologist time', while this could be beneficial in guiding future improvements. Recent findings suggest a more comprehensive approach to the total chain of care enabling rtPA treatment to eliminate bottlenecks in the entire pre- and intrahospital care pathway $[21,26,27]$. 
In conclusion, both DNT and the percentage of patients treated within $1 \mathrm{~h}$ after ED admission improved significantly, presumably through the combined impact of a series of structural and logistic interventions.

\section{Acknowledgements}

We would like to thank M. Scheele, A. van der Lugt and M. Eesteren for their contribution in finding the dates of all interventions. We received an Internal Erasmus MC grant for health care research (Mrace).

\section{References}

1 Hacke W, Donnan G, Fieschi C, Kaste M, von Kummer R, Broderick JP, et al: Association of outcome with early stroke treatment: pooled analysis of ATLANTIS, ECASS, and NINDS rt-PA stroke trials. Lancet 2004;363:768774.

-2 Wardlaw JM, Murray V, Berge E, del Zoppo G, Sandercock P, Lindley RL, et al: Recombinant tissue plasminogen activator for acute ischaemic stroke: an updated systematic review and meta-analysis. Lancet 2012;379: 2364-2372.

-3 Jauch EC, Saver JL, Adams HP Jr, Bruno A, Connors JJ, Demaerschalk BM, et al: Guidelines for the early management of patients with acute ischemic stroke: a guideline for healthcare professionals from the American Heart Association/American Stroke Association. Stroke 2013;44:870-947.

4 Desai JA, Smith EE: Prenotification and other factors involved in rapid tPA administration. Curr Atheroscler Rep 2013;15:337.

-5 Mikulik R, Kadlecova P, Czlonkowska A, Kobayashi A, Brozman M, Svigelj V, et al: Factors influencing in-hospital delay in treatment with intravenous thrombolysis. Stroke 2012;43:1578-1583.

6 Dirks M, Niessen LW, Huijsman R, van Wijngaarden J, Minkman MM, Franke CL, et al: Promoting Acute Thrombolysis for Ischaemic Stroke (PRACTISE). Int J Stroke 2007;2:151-159.

7 Dirks M, Niessen LW, van Wijngaarden JD, Koudstaal PJ, Franke CL, van Oostenbrugge RJ, et al: Promoting thrombolysis in acute ischemic stroke. Stroke 2011;42:1325-1330.

$\checkmark 8$ van der Wulp I, van Baar ME, Schrijvers AJ: Reliability and validity of the Manchester Triage System in a general emergency department patient population in the Netherlands: results of a simulation study. Emerg Med J 2008;25:431-434.

9 Glass G: Interrupted Time Series Quasi-Experiments. Complementary Methods for Research in Education, ed 2. Washington, American Educational Research Association, 1997, pp 589-609.

$\checkmark 10$ Iguchi Y, Kimura K, Sone K, Miura H, Endo H, Yamagata S, et al: Stroke incidence and usage rate of thrombolysis in a Japanese urban city: the Kurashiki stroke registry. J Stroke Cerebrovasc Dis 2013;22:349-357.

11 Singer OC, Hamann GF, Misselwitz B, Steinmetz H, Foerch C; Arbeitsgruppe Schlaganfall Hessen: Time trends in systemic thrombolysis in a large hospital-based stroke registry. Cerebrovasc Dis 2012;33:316-321.

12 Dalloz MA, Bottin L, Muresan IP, Favrole P, Foulon S, Levy P, et al: Thrombolysis rate and impact of a stroke code: a French hospital experience and a systematic review. J Neurol Sci 2012;314:120-125.

13 Bae HJ, Kim DH, Yoo NT, Choi JH, Huh JT, Cha JK, et al: Prehospital notification from the emergency medical service reduces the transfer and intra-hospital processing times for acute stroke patients. J Clin Neurol 2010; 6:138-142.

14 Casolla B, Bodenant M, Girot M, Cordonnier C, Pruvo JP, Wiel E, et al: Intra-hospital delays in stroke patients treated with rt-PA: impact of preadmission notification. J Neurol 2013;260:635-639.

15 Fassbender K, Balucani C, Walter S, LevineSR, Haass A, Grotta J: Streamlining of prehospital stroke management: the golden hour. Lancet Neurol 2013;12:585-596.

16 Helsinki Stroke Thrombolysis Registry Group: Does time of day or physician experience affect outcome of acute ischemic stroke patients treated with thrombolysis? A study from Finland. Int J Stroke 2012;7:511-516.

17 Kim SK, Lee SY, Bae HJ, Lee YS, Kim SY, Kang MJ, et al: Pre-hospital notification reduced the door-to-needle time for iv t-PA in acute ischaemic stroke. Eur J Neurol 2009;16:1331-1335.

18 Lau AY, Soo YO, Graham CA, Woo WK, Wong EH, Leung H, et al: An expedited stroke triage pathway: the key to shortening the door-to-needle time in delivery of thrombolysis. Hong Kong Med J 2010;16:455-462.

19 Lindsberg PJ, Happola O, Kallela M, Valanne L, Kuisma M, Kaste M: Door to thrombolysis: ER reorganization and reduced delays to acute stroke treatment. Neurology 2006;67:334-336.

20 Saver JL: Time is brain - quantified. Stroke 2006;37:263-266.

21 Baldereschi M, Piccardi B, Di Carlo A, Lucente G, Guidetti D, Consoli D, et al: Relevance of prehospital stroke code activation for acute treatment measures in stroke care: a review. Cerebrovasc Dis 2012;34:182-190. 
22 Griebe M, Kern R, Eisele P, Sick C, Wolf ME, Sauter-Servaes J, et al: Continuous magnetic resonance perfusion imaging acquisition during systemic thrombolysis in acute stroke. Cerebrovasc Dis 2013;35:554-559.

23 Tekle WG, Chaudhry SA, Hassan AE, Peacock JM, Lakshminarayan K, Tsai A, et al: Utilization of intravenous thrombolysis in 3-4.5 hours: analysis of the Minnesota stroke registry. Cerebrovasc Dis 2012;34:400-405.

-24 Schouten LM, Hulscher ME, van Everdingen JJ, Huijsman R, Grol RP: Evidence for the impact of quality improvement collaboratives: systematic review. BMJ 2008;336:1491-1494.

25 Meretoja A, Strbian D, Mustanoja S, Tatlisumak T, Lindsberg PJ, Kaste M: Reducing in-hospital delay to 20 min in stroke thrombolysis. Neurology 2012;79:306-313.

-26 Lahr MM, Luijckx GJ, Vroomen PC, van der Zee DJ, Buskens E: The chain of care enabling tPA treatment in acute ischemic stroke: a comprehensive review of organisational models. J Neurol 2013;260:960-968.

-27 Prabhakaran S, O’Neill K, Stein-Spencer L, Walter J, Alberts MJ: Prehospital triage to primary stroke centers and rate of stroke thrombolysis. JAMA Neurol 2013;70:1126-1132. 